\title{
Computed tomography and magnetic resonance imaging: past, present and future
}

\author{
N.L. Müller
}

Computed tomography and magnetic resonance imaging: past, present and future. N.L. Müller. (C) ERS Journals Ltd 2002.

ABSTRACT: The aims of this paper are to summarize the current recommendations for the use of computed tomography (CT) and magnetic resonance imaging (MRI) in the chest and to suggest some possible future developments.

The main developments of $\mathrm{CT}$ in the chest have been the introduction of highresolution CT (HRCT), spiral CT and, more recently, multidetector spiral CT.

HRCT is defined as thin-section CT (1- to 2-mm collimation scans), optimized by using a high-spatial resolution (edge-enhancing) algorithm. Several studies have shown that HRCT closely reflects macroscopic (gross) pathological findings. HRCT currently has the best sensitivity and specificity of any imaging method used for the assessment of focal and diffuse lung diseases.

The advent of spiral CT and, more recently, multidetector CT scanners, has allowed for major improvements in the imaging of airways, pulmonary and systemic vessels, and lung nodules. Spiral CT facilitates multiplanar and three-dimensional display of structures and visualization of pulmonary and systemic vessels, with a level of detail that is comparable to that of conventional angiography. With the use of graphics-based software programs, spiral CT enables depiction of the luminal surface of the airways with images that resemble those of bronchoscopy (virtual bronchoscopy) or bronchography (virtual bronchography). Several studies have shown a high sensitivity and specificity for spiral CT in the diagnosis of acute pulmonary embolism. Therefore, spiral CT is rapidly becoming the imaging modality of choice in the diagnosis of pulmonary embolism.

Like the radiograph, signal intensity on computed tomography is mainly due to a single parameter: electron density. The signal intensity of the magnetic resonance image depends on four parameters: nuclear density, two relaxation times called T1 and T2, and motion of the nuclei within the imaged lung volume. Abnormal soft tissue can be identified more easily through measurement of these four parameters than through use of computed tomography. Furthermore, because the spatial orientation of the image is determined by manipulation of magnetic fields, scans can be performed in any plane. The main indications for magnetic resonance in the chest have been in the evaluation of the heart, major vessels, mediastinum, and hilar structures because of the natural contrast provided by flowing blood. Of particular interest for the respirologist has been the recent development of magnetic resonance angiography. This technique consists of three-dimensional single breath-hold images obtained using gadolinium-based contrast agents. This is a promising technique for the diagnosis of acute and chronic pulmonary embolism.

Eur Respir J 2002; 19: Suppl. 35, 3s-12s.
Dept of Radiology, Vancouver General Hospital, University of British Columbia, Vancouver, Canada.

Correspondence: N.L. Müller Dept of Radiology

Vancouver General Hospital

University of British Columbia 855 West 12 th Ave

Vancouver

$\mathrm{BC}$

V5Z 1M9

Canada

Fax: 6048754319

E-mail: nmuller@vanhosp.bc.ca

Keywords: Chronic obstructive pulmonary diseases computed tomography interstitial lung disease lung computed tomography magnetic resonance imaging pulmonary embolism

Received: May 292001 Accepted May 302001
For many years, the plain chest radiograph was the only imaging modality used in the diagnosis of lung disease. With the advent of computed tomography (CT) came the first opportunity to assess gross lung structure. Conventional 8 - to $10-\mathrm{mm}$ collimation scans allowed better assessment of the lung parenchyma than was previously possible. However, CT only played a minor role in the diagnosis of interstitial and airway disease until the introduction of highresolution images in 1985. High-resolution CT (HRCT) has allowed major developments in understanding of the pathology and pathophysiology of diffuse lung diseases to take place. HRCT currently has the best sensitivity and specificity of any imaging method for the assessment of focal and diffuse lung diseases. The advent of spiral CT and, more recently, multidetector CT scanners has allowed for major improvements in the imaging of airways, pulmonary and systemic vessels, and lung nodules. Spiral CT facilitates multiplanar and three-dimensional (3D) display of structures and visualization of pulmonary and systemic vessels, with a level of detail comparable to that of conventional angiography. Through use of graphics-based software programs, spiral CT facilitates depiction of the luminal surface of the airways with images that resemble those of bronchoscopy (virtual bronchoscopy) or bronchography (virtual bronchography). 
Although CT provides superb morphological detail, it has limited contrast resolution and is therefore unable to distinguish tumour from inflammation or fibrosis. Magnetic resonance imaging (MRI) provides greater contrast resolution than CT. As a result, in many centres MRI has replaced CT in the assessment of the central nervous system and musculoskeletal abnormalities. MRI of the lungs has been limited by a low signal-to-noise ratio and by degradation of the image by motion artifacts. Recent studies, however, have suggested a potential role for MRI in lung disease.

The aims of this paper are to summarize some of the main current recommendations for the use of CT and MRI, highlight areas of controversy that require further study, and suggest some possible future developments.

\section{Computed tomography}

The main developments in CT in the chest have been the introduction of HRCT, spiral CT and, more recently, multidetector spiral $\mathrm{CT}$.

\section{High-resolution computed tomography}

A number of studies have shown that CT can play a major role in the assessment of patients who have diffuse lung disease. By eliminating superimposition of structures, CT allows for a better assessment of the type, distribution, and severity of parenchymal abnormalities than is possible with chest radiographs. It demonstrates both the normal and abnormal parenchyma down to the level of the secondary pulmonary lobules. Optimal assessment of parenchymal detail and small airways requires the use of HRCT. HRCT is defined as thin-section CT (1- to 2-mm collimation scans) optimized by using a high-spatial resolution (edge-enhancing) algorithm. Several studies have shown that high-resolution CT closely reflects the macroscopic (gross) pathological findings [1, 2]. Many parenchymal diseases preferentially affecting certain anatomical compartments can be visualized at lowpower microscopic evaluation [1-3]. These include diseases that are primarily broncho- or bronchiolocentric, angiocentric, perilymphatic, septal, diffuse interstitial and other distributions of disease that are also recognizable on HRCT [1-3]. By demonstrating the pattern and distribution of these abnormalities, HRCT often allows for a confident diagnosis to be made [4-6].

The clinical utility of HRCT can be assessed in terms of its sensitivity and specificity in the diagnosis of parenchymal and airway disease and its accuracy in the differential diagnosis of acute and chronic lung diseases.

Sensitivity and specificity of high-resolution computed tomography in the diagnosis of diffuse lung disease. Although the chest radiograph remains the first imaging modality used in the assessment of patients with suspected diffuse lung diseases, the radiograph is normal in $10-15 \%$ of patients with proven interstitial lung disease [7, 8]. Another limitation of chest radiographs is their relatively low specificity. Between $10-20 \%$ of patients interpreted as having diffuse parenchymal abnormalities on the radiograph have normal biopsy $[6,8]$. Several studies have shown that HRCT has greater sensitivity and specificity than the radiograph in the detection of diffuse lung disease [9-11]. Based on the data published in the literature, PADLEY et al. [6] concluded that in the detection of infiltrative lung disease, HRCT has a sensitivity of $\sim 94 \%$ and a specificity of $96 \%$, while the radiograph has a sensitivity of $80 \%$ and a specificity of $82 \%$. Based on these results, HRCT is indicated in patients with suspected diffuse infiltrative lung disease who have normal or questionable radiographic findings.

Diagnosis of bronchiectasis. HRCT is currently the imaging modality of choice for the diagnosis of bronchiectasis. In a study of 44 lungs in 36 patients, GrENIER et al. [12] demonstrated that compared to bronchography, HRCT had a sensitivity of $97 \%$ and a specificity of $93 \%$ in the diagnosis of bronchiectasis. Young et al. [13] assessed the diagnostic accuracy of HRCT compared to bronchography in 259 segmental bronchi from 70 lobes of 27 lungs. Bronchiectasis was correctly identified on HRCT in 87-89 segmental bronchi (sensitivity 97\%) and excluded in 169-170 segmental bronchi (specificity 99\%).

Accuracy in the differential diagnosis of chronic infiltrative lung diseases. It is often difficult to accurately characterize the abnormalities on chest radiographs because of the superimposition of structures and relatively low contrast resolution.

In an evaluation of 365 patients who had open-lung biopsy, which confirmed chronic infiltrative lung disease (CILD), McLoud et al. [14] found that their first two diagnostic choices corresponded to the histological diagnosis in only $50 \%$ of patients. Furthermore, there was only $70 \%$ interobserver agreement as to the predominant type of parenchymal abnormality or its extent.

MATHIESON et al. [4] compared the accuracy of conventional and HRCT with that of chest radiographs in making specific diagnoses in 118 consecutive patients who had chronic diffuse lung disease. The CT scans and radiographs were reviewed independently by three observers who listed their most likely diagnoses and their degree of confidence in these diagnoses. A confident diagnosis was reached more than twice as often with CT and HRCT than with chest radiographs $(49 \%$ and $23 \%$, respectively). More importantly, a correct diagnosis was made in $93 \%$ of the first-choice CT interpretations, as compared to $77 \%$ of the first-choice plain film interpretations $(\mathrm{p}<0.001)$.

GreniER et al. [5] confirmed the superior diagnostic accuracy of HRCT compared to radiographs in a study using HRCT with 1-mm images obtained every $10 \mathrm{~mm}$. The authors retrospectively evaluated 140 patients with CILD. Three independent observers made a correct first-choice diagnosis with radiographs 
in $64 \%$ of cases versus $76 \%$ with HRCT, regardless of the confidence level. When a confident first-choice diagnosis was made (defined as a $>75 \%$ chance of being correct), HRCT proved accurate in 53\% of cases, compared to $27 \%$ for chest radiographs $(\mathrm{p}<0.001)$.

In a subsequent study, GrenIER et al. [15] assessed the relative value of clinical data, chest radiographs, and HRCT scans in a retrospective analysis of 208 patients with CILD. A correct diagnosis was made based on clinical data alone in $29 \%$ of cases, radiography alone in $9 \%$, and HRCT in $36 \%$. This increased to $54 \%$ when clinical and radiographical findings were combined and $80 \%$ when all these were analysed together $(\mathrm{p}<0.01)$.

Accuracy in the differential diagnosis of acute lung disease. HRCT findings may also be of value in the diagnosis of acute lung disease in patients with acquired immune deficiency syndrome (AIDS) and immunocompromised non-AIDS patients. In a study of patients who had AIDS and acute lung disease, HARTMAN et al. [16] found that use of HRCT provided a correct first-choice diagnosis in $66 \%$ of the cases, regardless of the degree of confidence. A confident diagnosis was made in $48 \%$ of all cases and the observers were correct in $92 \%$ of those cases. The interpretations of CT scans were most often accurate in the confident diagnosis of Pneumocystis carinii pneumonia (94\%) and Kaposi's sarcoma (90\%). KANG et al. [17] compared CT to chest radiography in 89 patients who had a single proved thoracic complication. Two observers were confident in their firstchoice diagnoses in 61 of $178(34 \%)$ interpretations using chest radiographs and in 83 of $178(47 \%)$ interpretations using CT. The diagnosis was correct in $67 \%$ (41 of 61) of confident radiographical interpretations, compared to $87 \%$ (72 of 83 ) of interpretations at CT $(\mathrm{p}<0.01)$ [17].

CT has also been found to be superior to chest radiography in the differential diagnosis of acute pulmonary complications in immunocompromised non-AIDS patients [18]. In a study comparing CT and radiography's ability to detect and diagnose acute pulmonary complications in this group, the CT scans and radiographs of 45 immunocompromised nonAIDS patients who had proven pulmonary disease and 20 normal controls were independently assessed by two observers who had no knowledge of clinical or pathological data [18]. The sensitivity and specificity of the detection of pulmonary complications were $100 \%$ and $98 \%$, respectively, for CT, compared to $98 \%$ and $93 \%$, respectively, for chest radiography. In the immunocompromised patients, the first-choice diagnosis was correct in $44 \%$ of CT scans and $30 \%$ of radiograph readings $(\mathrm{p}<0.01)$. Confidence level one (definite) was reached in 33\% of CT scans and $10 \%$ of chest radiographs $(p<0.001)$. Diseases with a dominant nodular pattern had a higher occurrence of correct first-choice diagnoses (62\% versus $34 \%$; $\mathrm{p}<0.02)$ and level one confidence ratings $(53 \%$ versus $13 \% ; \mathrm{p}<0.001)$ than diseases with ground-glass opacity, consolidation, or irregular linear opacities [18].

HRCT can also be helpful in the differential diagnosis of acute parenchymal disease in immunocompetent patients. In a study of HRCT findings in 90 patients who had acute parenchymal diseases assessed without the benefit of clinical history, TomiYAma et al. [19] showed that two independent observers were $90 \%$ accurate in classifying diseases as infectious or noninfectious, although the types of infections studied were limited. Furthermore, the observers made a correct first-choice diagnosis in an average of 55 of 90 $(61 \%)$ cases. This included $50 \%$ of cases with bacterial pneumonia, 62\% with Mycoplasma pneumonia, 90\% with acute interstitial pneumonitis, $72 \%$ with hypersensitivity pneumonitis (HP), 30\% with acute eosinophilic pneumonia, and $28 \%$ with pulmonary haemorrhage.

Assessment of small airway disease. The term small airways disease is considered to be synonymous with bronchiolar disease. In many patients with bronchiolitis, a specific diagnosis can be suggested based on clinical history and the pattern and distribution of abnormalities on HRCT. The main abnormalities on HRCT consist of a tree-in-bud pattern, poorly defined centrilobular nodules and areas of decreased attenuation and air trapping [20].

The tree-in-bud pattern consists of well-defined centrilobular nodular and branching linear opacities, creating the appearance of a tree in bud [21, 22]. This pattern reflects the presence of either dilated bronchioles, filled with mucus or secretions, or bronchiolocentric nodules. In the vast majority of cases, a tree-in-bud pattern is due to acute or chronic infection, most commonly endobronchial spread of tuberculosis or viral or mycoplasma infection $[21,22]$. AQUINo et al. [21] identified a tree-in-bud pattern in $25 \%$ of patients who had bronchiectasis and had undergone HRCT (including patients with cystic fibrosis and allergic bronchopulmonary aspergillosis) and $17 \%$ of patients who had acute infectious bronchitis or pneumonia. This pattern was not identified in 141 HRCT studies of patients with noninfectious airway diseases, including emphysema, respiratory bronchiolitis (RB), constrictive bronchiolitis, bronchiolitis obliterans organizing pneumonia, and HP. Therefore, the presence of the tree-in-bud pattern is highly suggestive of an infectious cause of disease.

In the absence of intraluminal secretions, poorly defined centrilobular nodules usually indicate the presence of peribronchiolar inflammation. The most common causes of the bilateral symmetric centrilobular nodular pattern are HP, RB, and respiratory bronchiolitis-interstitial lung disease [23, 24].

Areas of decreased attenuation and air trapping indicate the presence of partial airway obstruction. Common causes include obliterative bronchiolitis and asthma [25, 26]. The presence of air trapping on expiratory HRCT is the most indicative finding in the diagnosis of obliterative bronchiolitis [26, 27]. In a study by LEUNG et al. [26], air trapping was found in 10 of 11 patients who had biopsy-diagnosed bronchiolitis obliterans (BO), compared to two of 10 patients who did not have biopsy-diagnosed BO or pulmonary function test (PFT) abnormalities [26]. Thus, air trapping was found to have a sensitivity of $91 \%$, a 
specificity of $80 \%$, and an accuracy of $86 \%$ for diagnosing $\mathrm{BO}$. However, patients with $\mathrm{BO}$ in this study already had established disease. The mean time from lung transplantation to $\mathrm{CT}$ in the study was 4.8 yrs and the mean duration of a known diagnosis of $\mathrm{BO}$ was $1.3 \mathrm{yrs}$.

In a study by LEE et al. [28], HRCT, including expiratory scans, was reviewed in consecutive normal lung transplant patients and patients first diagnosed as having $\mathrm{BO}$ or $\mathrm{BO}$ syndrome (BOS). The frequency of significant air trapping in patients who had $\mathrm{BO}$ or BOS was significantly higher than in patients who had a normal biopsy and PFTs. However, the sensitivity of significant air trapping on expiratory CT was only $74 \%$; its specificity was $67 \%$ and its accuracy $71 \%$.

BANKIER et al. [29] assessed the usefulness of evaluating air trapping on expiratory HRCT in the diagnosis of BOS. They performed 111 combined inspiratory and expiratory HRCT examinations in eight healthy control subjects and 38 heart-lung transplant recipients. The extent of air trapping increased with BOS severity $(\mathrm{p}=0.001)$. They found that a threshold of $32 \%$ of air trapping was optimal for distinguishing between patients with and without BOS, and provided a sensitivity of $83 \%$, a specificity of $89 \%$, and an accuracy of $88 \%$. The prevalence of BOS and positive predictive value of air trapping increased with postoperative time, but the negative predictive value of air trapping remained high throughout the study. BANKIER et al. [29] concluded that at a threshold of $32 \%$, air trapping is sensitive, specific, and accurate for diagnosing BOS, and that patients with air trapping below $32 \%$ are unlikely to have BOS [29].

\section{Spiral computed tomography}

Technique. A conventional CT scan of the chest consists of a series of cross-sectional slices obtained during suspended respiration. After each slice is obtained, the patient is allowed to breathe while the table is moved to the next scanning position. This method is known as incremental CT scanning. Although each image can be obtained in $\sim 1 \mathrm{~s}$, there is a delay of 5-10 s between the recording of images.

Spiral (helical) CT is an important technical advance, which allows continuous scanning while the patient is moved through the CT gantry, so that the $\mathrm{X}$-ray beam traces a helical or spiral curve in relation to the patient. Cross-sectional images can be reconstructed after the data specific to each plane of section has been estimated. This mathematical calculation is performed by interpolation of the spiral data above and below each plane of section.

Spiral CT scanners obtain high quality data through the entire volume of the chest. They therefore produce multiplanar reformations of the images and 3D image rendering. Multiplanar reformations are two-dimensional (2D) displays of any arbitrary plane in the imaged volume (fig. 1), which allow for better appreciation of the spatial relationships of various structures than a series of individual cross-sectional images. The most commonly used reformations are in
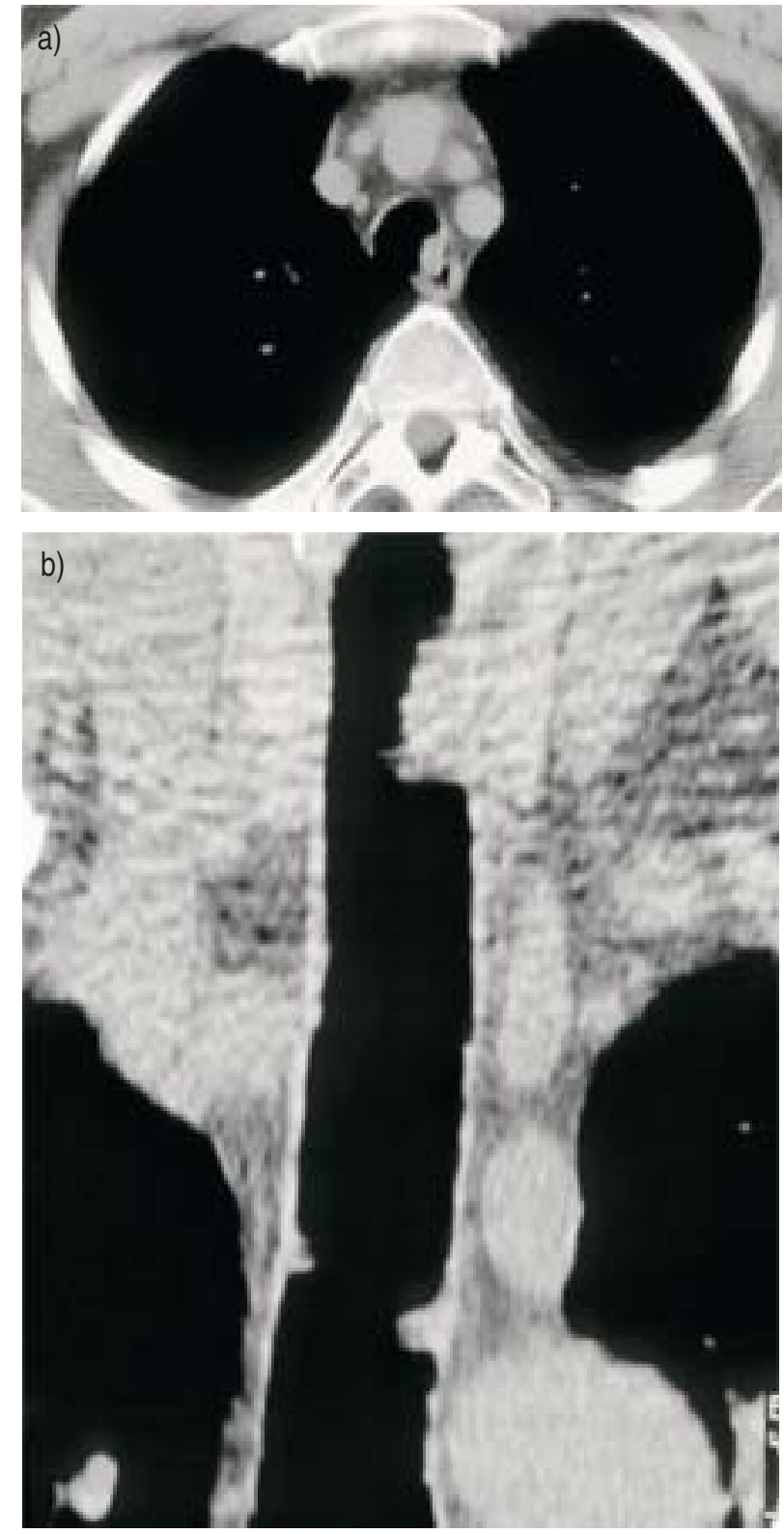

Fig. 1.-Value of multiplanar reconstructions on computed tomography (CT). a) Cross-sectional spiral CT image shows focal thickening of the tracheal wall. b) Coronal reconstruction demonstrates several focal abnormalities of the tracheal wall. The patient was shown at bronchoscopy to have multiple tracheal metastases.

the sagittal and coronal planes, although oblique and curved planar reconstructions are also possible. It has been shown that, compared to cross-sectional images, $2 \mathrm{D}$ reformations improve diagnostic accuracy when interpreting CT angiograms of pulmonary embolism (PE) [30]. This study showed improved detection of clot burden in cases of proven PE and more confident exclusion of clot in cases without PE.

The main techniques for the depiction of the entire volume of data are shaded-surface displays and true volumetric rendering [31, 32]. As the name implies, shaded-surface displays depict the surface of the selected anatomic structure and remove the remaining anatomy from the displayed image. Shaded-surface 
displays are optimal for structures that have large contrast differences with surrounding tissues, such as airways, vessels or bones. A range of attenuation values that identify the target tissue is selected (e.g. $-500-700$ Hounsfield units for pulmonary arteriovenous malformations) and the remaining image data is discarded. The object can be rotated and tipped to provide multiple viewing angles. Therefore, insights into the vascular anatomy of lesions that are not possible with pulmonary angiography, can be obtained with these displays. These images are also optimal for assessment of pulmonary vascular abnormalities, such as arteriovenous malformations (AVMs). Combined interpretation of surface shaded display images and the traditional transverse sections has been shown to provide complete pretherapeutic evaluation of pulmonary AVMs in $95 \%$ of cases [33]. True volume rendering displays structures of interest that are either brighter than adjacent structures (maximum intensity projection (MIP)) or less dense than adjacent structures (minimum intensity projection (MINIP)). The MIP and MINIP techniques remove any structure that does not have the same signal intensity as the selected structure. MIP images are ideal for depiction of vessels, calcification, stents and bones. MINIP images are ideal for the depiction of subtle areas of emphysema.

Use of graphics-based software systems and volume-rendering techniques allows depiction of the luminal surface of the airways, which resemble images seen by bronchography or during bronchoscopy [31, 32]. The CT technique that depicts the interior of the trachea and bronchi in a manner that resembles conventional bronchography is known as CT tracheobronchography. The technique that provides luminal surface views from the virtual environment of the CT database, which resemble those of bronchoscopy, is known as virtual bronchoscopy or, preferably, CT bronchoscopy.

Multidetector spiral computed tomography. The first four channel multidetector CT scanners were introduced in the late 1990s. The four rows can be used to give maximum spatial resolution or detector rows can be combined, decreasing spatial resolution but increasing the volume of tissue scanned per unit of time. As well as improvements in the detector array, the rotational velocity of the gantry was increased from $1 \mathrm{~s}$ in single section spiral CT scanners to $0.5 \mathrm{~s}$ in the fastest multidetector spiral CT scanners. The combination of four read-out channels and twice the rotational speed increased the scanning speed to eight times higher than that of single section spiral CT equipment. These improvements in data-acquisition speed can be used in CT angiography to increase the volume scanned or to scan the same volume using thinner sections. The use of thinner sections decreases volume averaging and allows visualization of smaller vessels. It has been shown that four row multisection CT scanners can routinely identify subsegmental pulmonary artery segments [34, 35]. Faster gantry rotation time decreases the effect of cardiac motion on noncardiac-gated acquisitions. In addition, the combination of faster gantry rotation time, simultaneous acquisition of the patient's electrocardiogram and specialized reconstruction techniques can provide almost motion-free images during cardiac systole or diastole [36]. Cardiac-gated images should facilitate the calculation of cardiac volumes and ejection fraction using CT.

The first eight channel multidetector CT scanners were announced in November 2001.

Spiral computed tomography in pulmonary embolism. Several studies have shown a high sensitivity and specificity for spiral CT in the diagnosis of $P E$ [37-39]. In an increasing number of centres, spiral CT has become the imaging modality of choice in the diagnosis of PE. Characteristic findings of acute $\mathrm{PE}$ on spiral CT consist of partial central or marginal filling defects surrounded by a thin rim of contrast material or complete filling defect with obstruction of an entire vessel section.

Diagnostic accuracy of spiral computed tomography. The reported diagnostic accuracy of spiral CT has varied depending on the technique used, the patient population, and whether the authors limited the analysis of the central pulmonary arteries to the level of the segmental vessels or included subsegmental arteries. Overall, studies have shown a spiral CT sensitivity of $90 \%$, a specificity of $90 \%$, a positive predictive value of $93 \%$ and a negative predictive value of $94 \%$ for emboli, down to and including the level of the segmental pulmonary arteries [40]. Studies comparing spiral CT with ventilation/perfusion scintigraphy consistently demonstrated that spiral CT had a greater diagnostic accuracy [37, 39, 41]. In addition, these studies consistently showed better interobserver agreement in the interpretation of spiral CT images than ventilation/perfusion scans. It has also been shown that patients who have a negative spiral CT and are not anticoagulated are unlikely to have evidence of PE on clinical follow-up [42, 43]. One group of investigators assessed patient outcome after a clinical follow-up of a minimum of 6 months, in 78 consecutive patients in whom spiral CT scans were interpreted as negative for pulmonary thromboembolism (PTE) and anticoagulant therapy was not administered [42]. Nine patients died, one of whom was shown to have a 1- to 2-mm embolus at autopsy performed 7 days after CT scan, which may have been missed on spiral CT and angiography. No evidence of PTE was found in any of the other 77 patients. The negative predictive value for spiral CT was $99 \%$ in this study [42].

\section{Magnetic resonance imaging}

Although CT is more sensitive than radiography in detecting lung disease, like radiography, CT has a limited ability to differentiate between fibrosis and active inflammation, tumour and oedema. As a result of the increased soft-tissue contrast, magnetic resonance (MR) is superior to $\mathrm{CT}$ in distinguishing various tissue characteristics. It is therefore helpful in the distinction of radiation fibrosis from tumour 
recurrence in bronchogenic carcinoma and residual or recurrent lymphoma from fibrosis [44, 45]. It also allows distinction of obstructive from nonobstructive atelectasis [46].

The intensity of the MR image depends on four parameters: nuclear density, two relaxation times, called T1 and T2, and motion of the nuclei within the imaged lung volume. As the nuclear density increases, increasing numbers of nuclei align with the magnetic field, producing a more intense MR signal. The T1 relaxation time is a time constant in which the nuclei align in a given magnetic field or the magnetic vector along the direction of the applied field is re-established after a perturbation. T2 is the time constant for loss of phase coherence of excited spins. Signal is also influenced by motion, e.g. blood flow. Abnormal soft tissue can be better differentiated through measurement of these four parameters than through any other previous technique. In addition, scans can be performed in any plane, because the spatial orientation of the image is determined by manipulation of magnetic fields. As a result, MRI has become the best imaging method for the evaluation of neurological and musculoskeletal structures. It has also been used in the chest to evaluate the heart, major vessels, mediastinum and hilar structures because of the natural contrast provided by flowing blood.

Clinical application of MRI in the assessment of the lung parenchyma has been inhibited by physiological motion, which severely reduces image quality. Accordingly, as with early CT scanning, the clinical application of MRI in the thorax came much later than its use in other regions of the body.

Currently, the main indications for MRI in the chest are for evaluation of the heart and great vessels, the mediastinum and hila, and the chest wall. Of particular interest to respirologists has been the recent development of MR angiography. This technique consists of 3D single breath-hold images obtained using gadolinium-based contrast agents. Depending on the technique used, blood has a high (white blood angiography) or low signal intensity (black blood angiography) on MR angiography. White blood angiography is ideal for the depiction of flowing blood or pulmonary emboli, while black blood angiography is ideal for the depiction of the vessel wall.

\section{Evaluation of the heart and great vessels}

MRI has a well-established role in the assessment of congenital abnormalities of the heart and great vessels. It is superior to echocardiography in the assessment of adult congenital heart disease because it permits unobstructed views of all atrial, ventricular, and great vessel abnormalities [47, 48]. However, it is usually reserved for patients who have nondiagnostic or equivocal findings on echocardiography [48]. MR also allows excellent evaluation of central pulmonary artery abnormalities. Cine gradient-echo sequences permit assessment of cardiac wall motion and can detect high velocity jets related to ventricular septal defects, valvular regurgitation, or focal stenoses [47, 49].
Velocity-encoded cine sequences can be used to calculate blood flow [50].

Although comparable to conventional CT in the assessment of aortic aneurysms and aortic dissection, MRI has been shown to be inferior to spiral CT [51]. In one study, in which spiral CT, multiplanar transoesophageal echocardiography and MRI were compared, all three techniques were found to have $100 \%$ sensitivity in the detection of thoracic aortic dissection, but spiral CT had a higher specificity [51].

\section{Evaluation of the mediastinum and hila}

Currently, MRI is the secondary imaging modality in the evaluation of the mediastinum and hila, and is mainly used as a problem-solving technique in cases in which CT findings are equivocal. MRI has been shown to be superior to $\mathrm{CT}$ in the assessment of mediastinal and vascular invasion in patients who have pulmonary carcinoma [52]. It can also be helpful in the diagnosis of bronchogenic cysts in cases where CT findings are not diagnostic. These lesions characteristically show a homogeneous high signal intensity on T2-weighted MR images as a result of their fluid content (fig. 2) [53].

\section{Evaluation of the chest wall}

MRI allows excellent assessment of primary chest wall tumours and chest wall extension by lymphoma and pulmonary carcinoma, particularly those located in the superior sulcus region [54-56]. Because it provides excellent assessment of the tissue characteristics of the mass and the presence or absence of intraspinal extension, MRI is also ideal for the evaluation of neurogenic tumours [57, 58]. The technique is therefore the imaging modality of choice in the assessment of paraspinal lesions.

\section{Evaluation of pulmonary embolism}

As with CT, MRI allows direct visualization of pulmonary emboli. It has the additional advantage of not requiring radiation or the use of iodinated intravenous contrast, but is more expensive and less readily available. Other disadvantages of MRI include cardiac and respiratory motion artifacts and complex pulmonary blood flow patterns that may mimic embolism. These problems have been minimized through the development of gradient-recalled echo (GRE) imaging techniques obtained during a single breath hold. The combination of these techniques with gadolinium enhancement results in good quality MR angiographic images of the pulmonary arteries.

The potential usefulness of MR has been shown by several investigators. In one study of 30 consecutive patients who had suspected PTE, MR angiography was performed during the pulmonary arterial phase of an intravenous bolus of gadolinium [59]. The procedure was carried out using a coronal 3D gradient-echo pulse sequence with a slice thickness 

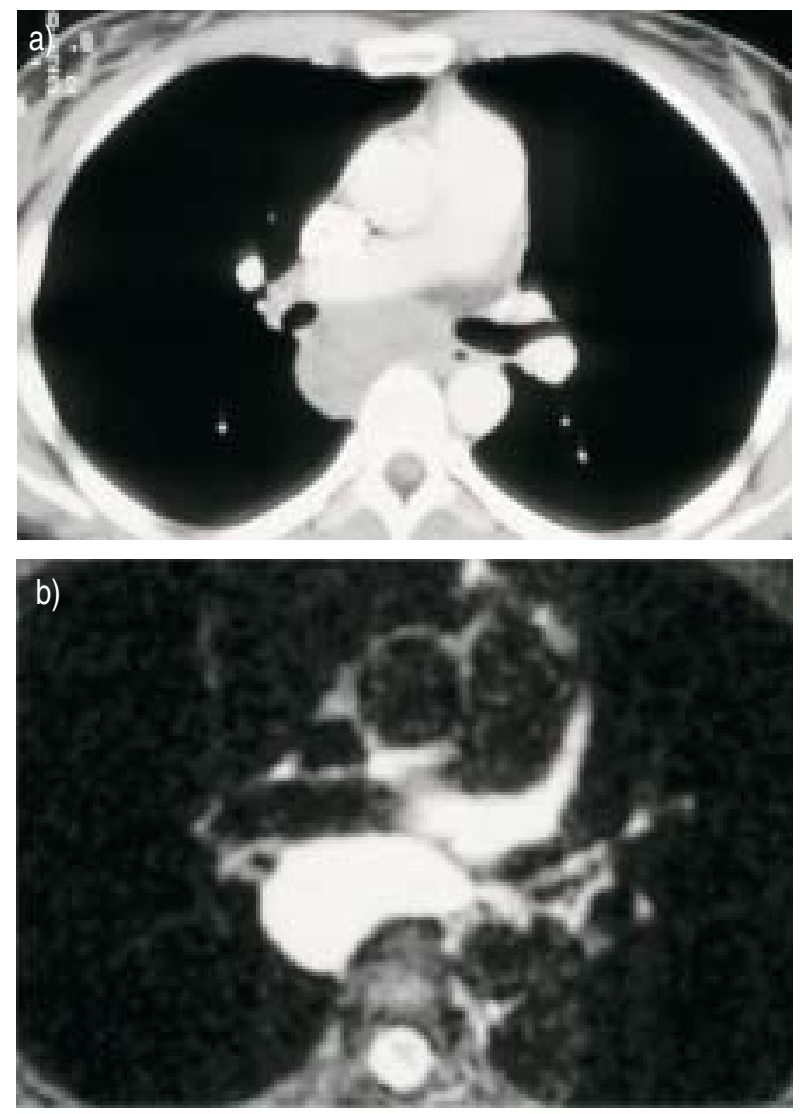

Fig. 2.-Superior contrast resolution of magnetic resonance (MR) as compared to computed tomography (CT). a) CT demonstrates subcarinal mass. This has attenuation values similar to those of soft tissue. b) T2-weighted MR image shows that the mass has homogeneous high signal intensity, similar to that of cerebrospinal fluid in the spinal canal. This appearance is characteristic of bronchogenic cyst. The soft tissue attenuation on CT is due to the presence of proteinaceous material within the cyst.

of $3-4 \mathrm{~mm}$ and an imaging time of $27 \mathrm{~s}$. The images were reviewed by three independent observers and the results compared to those of standard pulmonary angiography. All five lobar emboli and 16 of 17 segmental emboli identified on conventional pulmonary angiography were also identified on MR angiography. Two of the three observers reported one false-positive MR angiogram. Using conventional angiography as the gold standard, the three observers had diagnostic sensitivities of 100,87 , and $75 \%$ and specificities of 95,100 , and $95 \%$, respectively.

In a second investigation of 36 consecutive patients, MR angiography was performed during suspended respiration and the pulmonary arterial phase of a gadolinium-based contrast medium injection using a steady-state GRE sequence [60]. The standard MR images and coronal MIP images were reviewed on a computer workstation. Using digital subtraction angiography as the gold standard, PTE was correctly identified on MRI in 11 of 13 patients $(85 \%$ sensitivity) and excluded in 22 of 23 patients $(96 \%$ specificity). Both cases of false-negative interpretation were in patients who had only small subsegmental emboli.

\section{Lung parenchyma}

Experimental studies have suggested that MRI can be used to quantitate lung water content [61, 62]. Using animal models, close agreement has been shown between MRI signal intensities and gravimetrically determined lung wet-to-dry weight ratios. Although determination of absolute lung water content has proven difficult in vivo, measurements of relative lung water changes have been shown to be proportional to true lung water content and may prove to be a sensitive method for following the course of lung injury [63].

MAYO et al. [62] demonstrated that lung water and pleural pressure gradients can be assessed using MR. They imaged five normal volunteers in the supine and prone positions during quiet breathing and in the supine position at total lung capacity (TLC), using a cardiac-gated multi-echo pulse sequence with echo spacing of $20 \mathrm{~ms}$ from TE20 to $240 \mathrm{~ms}$ on a Picker $0.15 \mathrm{~T}$ MR imager (Picker Industries, Cleveland, Ohio, USA). After validating the technique, they demonstrated that there was no significant difference in average lung density in the prone $(0.21 \pm$ $\left.0.03 \mathrm{~g} \cdot \mathrm{mL}^{-1}\right)$ and supine $\left(0.20 \pm 0.03 \mathrm{~g} \cdot \mathrm{mL}^{-1}\right)$ positions. Lung density decreased at TLC $\left(0.12 \pm 0.01 \mathrm{~g} \cdot \mathrm{mL}^{-1}\right.$; $\mathrm{p}<0.01)$. Gradients in lung density were visible in all prone and supine scans at functional residual capacity and, on average, the gradients decreased by $90 \%$ at TLC.

MülLER et al. [64] compared the value of MRI to HRCT in the assessment of chronic infiltrative lung disease in 25 patients. All patients had cardiac-gated spin-echo MRI and $1.5 \mathrm{~mm}$ collimation HRCT. HRCT was considerably better than MRI in the anatomic assessment of the lung parenchyma and in demonstrating fibrosis. However, areas of airspace opacification (ground-glass attenuation and parenchymal consolidation) were equally well seen on MR as on HRCT. Open lung biopsy demonstrated that the areas of airspace opacification seen on MRI and HRCT corresponded to areas of active alveolitis or airspace infiltrates. Follow-up demonstrated similar degrees of change in the airspace opacification over time on MRI and CT. The authors concluded that whereas MRI was considerably inferior to HRCT in the initial assessment of patients with chronic infiltrative lung disease, it may potentially play a role in the assessment and follow-up of patients with predominantly airspace opacification.

Primack et al. [65] compared the MRI with the pathological findings in 22 consecutive patients with chronic infiltrative lung disease. They demonstrated that the MRI findings correlated closely with the macroscopic findings on open lung biopsy. Evidence of parenchymal opacification was seen on MRI in 14 patients, nine were interpreted as being equivalent to ground-glass intensity and five to consolidation. In 12 of the 14 patients the parenchymal opacification represented an active inflammatory process including alveolitis, pneumonia, and granulomatous infection, whereas in two patients it represented fibrosis. As the authors pointed out, a major limitation of MR in the assessment of infiltrative lung disease is the low spatial 
resolution compared to HRCT. Therefore, MR is not recommended in the routine assessment of patients with infiltrative lung disease. It may be used as an alternative imaging modality in patients who express concern about radiation exposure or in young patients who are repeatedly scanned for assessment of response to treatment or disease progression [65].

\section{Recent developments in chest magnetic resonance imaging}

Several recent developments promise to revolutionize the evaluation of parenchymal, airway, pulmonary vascular and chest wall abnormalities with MRI. These include breath-hold MRI, MR fluoroscopy, velocity encoded MR and hyperpolarized ${ }^{3} \mathrm{He}-$ enhanced MRI [66-70].

Velocity-encoded MRI allows accurate and direct measurement of the stroke volume of the right and left ventricles, pulmonary blood flow and pulmonary vascular resistance [69]. This technique may aid understanding of the pathophysiological changes within the pulmonary circulation in patients with pulmonary parenchymal disease or pulmonary vascular abnormalities.

Hyperpolarized ${ }^{3} \mathrm{He}$-enhanced MRI allows assessment of regional ventilation [70]. This technique may provide new insights into understanding of regional ventilation in patients with various airway abnormalities. Other gases that have been used for direct imaging of pulmonary ventilation on MRI include ${ }^{129} \mathrm{Xe},{ }^{19} \mathrm{~F}$, and oxygen.

Magnetic resonance's ability to provide threedimensional reconstructions and functional quantification can be applied to the assessment of the lung parenchyma, pulmonary vessels, airways, and the chest wall. Breath-hold magnetic resonance allows measurement of lung volumes and evaluation of diaphragmatic and chest wall mechanics $[66,68]$.

\section{References}

1. Müller NL, Miller RR. State-of-the-Art: computed tomography of chronic diffuse infiltrative lung disease. Part 1. Am Rev Respir Dis 1990; 142: 1206-1215.

2. Müller NL, Miller RR. State-of-the-Art: computed tomography of chronic diffuse infiltrative lung disease. Part 2. Am Rev Respir Dis 1990; 142: 1440-1448.

3. Colby TV, Swensen SJ. Anatomic distribution and histopathologic patterns in diffuse lung disease: correlation with HRCT. J Thorac Imaging 1996; 11: $1-26$.

4. Mathieson JR, Mayo JR, Staples CA, et al. Chronic diffuse infiltrative lung disease: comparison of diagnostic accuracy of CT and chest radiography. Radiology 1989; 171: 111-116.

5. Grenier P, Valeyre D, Cluzel P, et al. Chronic diffuse interstitial lung disease: diagnostic value of chest radiography and high-resolution CT. Radiology 1991; 179: 123-132.

6. Padley SPG, Hansell DM, Flower CDR, et al. Comparative accuracy of high resolution computed tomography and chest radiography in the diagnosis of chronic diffuse infiltrative lung disease. Clin Radiol 1991; 44: 222-226.

7. Epler GR, McLoud TC, Gaensler EA, et al. Normal chest roentgenograms in chronic diffuse infiltrative lung disease. N Engl J Med 1978; 298: 801-809.

8. Gaensler EA, Carrington CB. Open biopsy for chronic diffuse infiltrative lung disease: clinical, roentgenographic, and physiologic correlations in 502 patients. Ann Thorac Surg 1980; 30: 411-426.

9. Stein MG, Mayo J, Müller N, et al. Pulmonary lymphangitic spread of carcinoma: appearance on CT scans. Radiology 1987; 162: 371-375.

10. Müller NL, Kullnig P, Miller RR. The CT findings of pulmonary sarcoidosis: analysis of 25 patients. AJR 1989; 152: 1179-1182.

11. Müller NL. Clinical value of high-resolution CT in chronic diffuse lung disease. AJR 1991; 157: 11631170.

12. Grenier P, Maurice F, Musset D, et al. Bronchiectasis: assessment by thin-section CT. Radiology 1986; 161: 95-99.

13. Young K, Aspestrand F, Kolbenstvedt A. High resolution CT and bronchography in the assessment of bronchiectasis. Acta Radiol 1991; 32: 439-441.

14. McLoud TC, Carrington CB, Gaensler EA. Diffuse infiltrative lung disease: a new scheme for description. Radiology 1983; 149: 353-363.

15. Grenier P, Chevret S, Beigelman C, et al. Chronic diffuse infiltrative lung disease: determination of the diagnostic value of clinical data, chest radiography, and CT with Bayesian analysis. Radiology 1994; 191: 383-390.

16. Hartman TE, Primack SL, Müller NL, et al. Diagnosis of thoracic complications in AIDS: accuracy of CT. AJR 1994; 162: 547-553.

17. Kang EY, Staples CA, McGuinness G, et al. Detection and differential diagnosis of pulmonary infections and tumors in patients with AIDS: value of chest radiography versus CT. AJR 1996; 166: 15-19.

18. Janzen DL, Padley SP, Adler BD, et al. Acute pulmonary complications in immunocompromised non-AIDS patients: comparison of diagnostic accuracy of CT and chest radiography. Clin Radiol 1993; 47: $159-165$.

19. Tomiyama N, Müller NL, Johkoh T, et al. Acute parenchymal lung disease in immunocompetent patients: diagnostic accuracy of high-resolution CT. AJR 2000; 174: 1745-1750.

20. Müller NL, Miller RR. State-of-the-Art. Diseases of the bronchioles: CT and histopathologic findings. Radiology 1995; 196: 3-12.

21. Aquino SL, Gamsu G, Webb WR, et al. Tree-in-bud pattern: frequency and significance on thin-section CT. J Comput Assist Tomogr 1996; 20: 594-599.

22. Franquet T, Stern EJ. Bronchiolar inflammatory diseases: high-resolution CT findings with histologic correlation. Eur Radiol 1999; 9: 1290-1303.

23. Remy-Jardin M, Remy J, Wallaert B, Müller NL. Subacute and chronic bird breeder hypersensitivity pneumonitis: sequential evaluation with $\mathrm{CT}$ and correlation with lung function tests and bronchoalveolar lavage. Radiology 1993; 198: 111-118.

24. Heyneman LE, Ward S, Lynch DA, Remy-Jardin M, Johkoh T, Müller NL. Respiratory bronchiolitis, respiratory bronchiolitis associated interstitial lung disease, and desquamative interstitial pneumonia: 
different entities or part of the spectrum of the same disease process? AJR 1999; 173: 1617-1622.

25. Worthy SA, Müller NL, Hartman TE, Swensen SJ, Padley SPG, Hansell DM. Mosaic attenuation pattern on thin-section CT scans of the lung: differentiation among infiltrative lung, airway, and vascular diseases as a cause. Radiology 1997; 205: 465-470.

26. Leung AN, Fisher K, Valentine V, et al. Bronchiolitis obliterans after lung transplantation: detection using expiratory HRCT. Chest 1998; 113: 365-370.

27. Worthy SA, Park CS, Kim JS, Müller NL. Bronchiolitis obliterans after lung transplantation: highresolution CT findings in 15 patients. AJR 1997; 169: 673-677.

28. Lee ES, Gotway MB, Reddy GP, Golden JA, Keith FM, Webb WR. Early bronchiolitis obliterans following lung transplantation: accuracy of expiratory thinsection CT for diagnosis. Radiology 2000; 216: 472 477.

29. Bankier AA, van Muylem A, Knoop C, Estenne M, Gevenois PA. Bronchiolitis obliterans syndrome in heart-lung transplant recipients: diagnosis with expiratory CT. Radiology 2001; 218: 533-539.

30. Remy-Jardin M, Remy J, Cauvain O, Petyt L, Wannebroucq J, Beregi J. Diagnosis of central pulmonary embolism with helical CT: role of two dimensional multiplanar reformations. AJR 1995; 165: 1131-1138.

31. Remy-Jardin M, Remy J, Artaud D, et al. Volume rendering of the tracheobronchial tree: clinical evaluation of bronchographic images. Radiology 1998; 208: $761-770$.

32. Higgins WE, Ramaswamy K, Swift RD, et al. Virtual bronchoscopy for three-dimensional pulmonary image assessment: State of the art and future needs. Radiographics 1998; 18: 761.

33. Remy J, Remy-Jardin M, Giraud F, Wattinne L. Angioarchitecture of pulmonary arteriovenous malformations: clinical utility of three-dimensional helical CT. Radiology 1994; 191: 657-664.

34. Ghaye B, Szapiro D, Delannoy V, Mastora I, Remy J, Remy-Jardin M. How far does multidetector CT allow the analysis of peripheral pulmonary arteries? A comparative study with thin collimation single detector CT. Radiology 2000; 217: 508 .

35. Schoepf UO, Holzknecht NG, Helmberger TK, Hong CS, Huber AM, Reiser MF. Segmental and subsegmental pulmonary embolism (PE): improved detection with thin-slice multi-detector array spiral CT (MDCT). Radiology 2000; 217: 509.

36. Ohnesorge B, Flohr T, Becker C, et al. Cardiac imaging by means of electrocardiographical gated multisection spiral CT: initial experience. Radiology 2000; 217: 564-571.

37. Remy-Jardin M, Remy J, Deschildre F, et al. Diagnosis of pulmonary embolism with spiral CT: Comparison with pulmonary angiography and scintigraphy. Radiology 1996; 200: 699-706.

38. van Rossum AB, Pattynama PMT, Tjin A, et al. Pulmonary embolism: validation of spiral CT angiography in 149 patients. Radiology 1996; 201: 467-470.

39. Mayo JR, Remy-Jardin M, Müller NL, et al. Pulmonary embolism: prospective comparison of spiral CT with ventilation-perfusion scintigraphy. Radiology 1997; 205: 447-452.

40. Maki DD, Gefter WB, Alavi A. Recent advances in pulmonary imaging. Chest 1999; 116: 1388-1402.
41. Blachere H, Latrabe V, Montaudon M, et al. Pulmonary embolism revealed on helical CT angiography: comparison with ventilation-perfusion radionuclide lung scanning. AJR 2000; 174: 1041-1047.

42. Garg K, Sieler H, Welsh CH, Johnston RJ, Russ PD. Clinical validity of helical CT being interpreted as negative for pulmonary embolism: implications for patient treatment. AJR 1999; 172: 1627-1631.

43. Goodman LR, Lipchik RJ, Kuzo RS, Liu Y, McAuliffe TL, O'Brien DJ. Subsequent pulmonary embolism: risk after a negative helical CT pulmonary angiogram - prospective comparison with scintigraphy. Radiology 2000; 215: 535-542.

44. Glazer HS, Lee JKT, Levitt RL, et al. Radiation fibrosis: differentiation from recurrent tumor by MR imaging. Radiology 1985; 156: 721-726.

45. Nyman R, Rehn S, Glimelius B, et al. Magnetic resonance imaging for assessment of treatment effects in mediastinal Hodgkin's disease. Acta Radiol Diagn 1987; 28: 145-151.

46. Herold CJ, Kuhlman JE, Zerhouni EA. Pulmonary atelectasis: signal patterns with MR imaging. Radiology 1991; 178: 715-720.

47. Higgins CB, Sakuma H. Heart disease: Functional evaluation with MR imaging. Radiology 1996; 199: 307-315.

48. Higgins $\mathrm{CB}$, Caputo GR. Role of MR imaging in acquired and congenital cardiovascular disease. $A J R$ 1993; 161: 13-22.

49. Sechtem U, Pflugfelder PW, White RD, et al. Cine MR imaging: potential for the evaluation of cardiovascular function. AJR 1987; 148: 239-246.

50. Kondo C, Caputo GR, Semelka R, et al. Right and left ventricular stroke volume measurements with velocity-encoded cine MR imaging: in vitro and in vivo validation. AJR 1991; 157: 9-16.

51. Sommer T, Fehske W, Holzknecht N, et al. Aortic dissection: a comparative study of diagnosis with spiral CT, multiplanar transesophageal echocardiography, and MR imaging. Radiology 1996; 199: 347352 .

52. Webb WR, Gatsonis C, Zerhouni EA, et al. CT and MR imaging in staging non-small cell bronchogenic carcinoma: report of the radiologic diagnostic oncology group. Radiology 1991; 178: 705-713.

53. Nakata $\mathrm{H}$, Egashira $\mathrm{K}$, Watanabe $\mathrm{H}$, et al. MRI of bronchogenic cysts. J Comput Assist Tomogr 1993; 17: 267-270.

54. Fortier MV, Mayo JR, Swensen SJ, et al. MR imaging of chest wall lesions. Radiographics 1994; 14: 597-606.

55. Bergin CJ, Healy MV, Zincone GE, et al. MR evaluation of chest wall involvement in malignant lymphoma. J Comp Assist Tomogr 1990; 14: 928-932.

56. Heelan RT, Demas BE, Caravelli JF, et al. Superior sulcus tumors: CT and MR imaging. Radiology 1989; 170: 637-641.

57. Flickinger FW, Yuh WT, Behrendt DM. Magnetic resonance imaging of mediastinal paraganglioma. Chest 1988; 94: 652-654.

58. Siegel MJ, Jamroz GA, Glazer HS, et al. MR imaging of intraspinal extension of neuroblastoma. $J$ Comput Assist Tomogr 1986; 10: 593-595.

59. Meaney JFM, Weg JG, Chenevert TL, Stafford-Johnson D, Hamilton BH, Prince MR. Diagnosis of pulmonary embolism with magnetic resonance angiography. New Engl J Med 1997; 336: 1422-1427.

60. Gupta A, Frazer CK, Ferguson JM, et al. Acute 
pulmonary embolism: diagnosis with MR angiography. Radiology 1999; 210: 353-359.

61. Mayo JR, Müller NL, Forster BB, Okazawa M, Paré PD. Magnetic resonance imaging of hydrostatic pulmonary edema in isolated dog lungs: comparison with computed tomography. Can Assoc Radiol J 1990; 41: 281-286.

62. Mayo JR, MacKay AL, Whittall KP, Baile EM, Paré PD. Measurement of lung water content and pleural pressure gradient with magnetic resonance imaging. J Thorac Imaging 1995; 10: 73-81.

63. Cutillo AG, Morris AH, Ailion DC, Dureny $\mathrm{CH}$. Clinical implications of nuclear magnetic resonance lung research. Chest 1989; 96: 643-652.

64. Müller NL, Mayo JR, Zwirewich CV. Value of MR imaging in the evaluation of chronic infiltrative lung diseases: comparison with CT. AJR 1992; 158: 12051209.

65. Primack SL, Mayo JR, Hartman TE, Miller RR, Müller NL. MRI of infiltrative lung disease: comparison with pathologic findings. $J$ Comput Assist Tomogr 1994; 18: 233-238.
66. Qanadli SD, Orvoen-Frija E, Lacombe P, Di Paola R, Bittoun J, Frija G. Estimation of gas and tissue lung volumes by MRI: functional approach of lung imaging. J Comput Assist Tomogr 1999; 23: 743-748.

67. Holland AE, Goldfarb JW, Edelman RR. Diaphragmatic and cardiac motion during suspended breathing: preliminary experience and implications for breathhold MR imaging. Radiology 1998; 209: 483-489.

68. Cluzel P, Similowski T, Chartrand-Lefebvre C, Zelter M, Derenne J-P, Grenier PA. Diaphragm and chest wall: assessment of the inspiratory pump with MR imaging - preliminary observations. Radiology 2000; 215: 574-583.

69. Mousseaux E, Tasu JP, Jolivet O, Simonneau G, Bittoun J, Gaux J-C. Pulmonary arterial resistance: noninvasive measurement with indexes of pulmonary flow estimated at velocity-encoded MR imaging preliminary experience. Radiology 1999; 212: 896-902.

70. McAdams HP, Palmer SM, Donnelly LF, Charles HC, Tapson VF, MacFall JR. Hyperpolarized 3Heenhanced MR imaging of lung transplant recipients: preliminary results. AJR 1999; 173: 955-959. 